\title{
Diffraction and microscopy with attosecond electron pulse trains
}

\author{
Y. Morimoto ${ }^{1,2}$ and P. Baum ${ }^{1,2, *}$ \\ ${ }^{1}$ Ludwig-Maximilians-Universität München, Am Coulombwall 1, 85748 Garching, Germany \\ ${ }^{2}$ Max-Planck-Institute of Quantum Optics, Hans-Kopfermann-Str. 1, 85748 Garching, Germany \\ *peter.baum@lmu.de
}

Attosecond spectroscopy ${ }^{1-7}$ can resolve electronic processes directly in time, but a movielike space-time recording is impeded by the too long wavelength ( $\sim 100$ times larger than atomic distances) or the source-sample entanglement in re-collision techniques ${ }^{8-11}$. Here we advance attosecond metrology to picometer wavelength and sub-atomic resolution by using free-space electrons instead of higher-harmonic photons $s^{1-7}$ or re-colliding wavepackets ${ }^{8-11}$. A beam of 70-keV electrons at $4.5 \mathrm{pm}$ de Broglie wavelength is modulated by the electric field of laser cycles into a sequence of electron pulses with sub-optical-cycle duration. Timeresolved diffraction from crystalline silicon reveals a $<10$-as delay of Bragg emission and demonstrates the possibility of analytic attosecond-Angstrom diffraction. Real-space, lowmagnification electron microscopy visualizes with sub-light-cycle resolution how an optical wave propagates in space and time. This unification of attosecond science with electron microscopy and diffraction will enable space-time imaging of light-driven processes in the entire range of sample morphologies that electron microscopy can access.

Our concepts for (a) generating attosecond electron pulses, (b) direct streaking-oscilloscope characterization, (c) atomic diffraction and (d) proof-of-principle attosecond electron microscopy

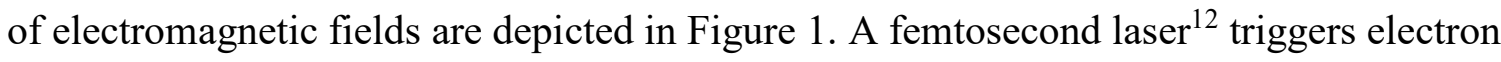
emission from a photocathode (yellow) and produces electron pulses of $\sim 1$ ps duration at a 
central energy of $E_{e l}=70 \mathrm{keV}^{13}$. The de Broglie wavelength is $\lambda_{e l} \approx 4.5 \mathrm{pm}$ and space charge effects are avoided by using less than one electron per pulse at the sample. A first laser beam ("modulation") is used to compress the electron pulse into a sub-cycle pulse train. For this purpose, we let the laser beam and the electron beam intersect at a 50-nm-thick silicon nitride membrane which lets the electrons pass through. The membrane is also transparent to the laser beam (1030 nm wavelength), but the refractive index of $\sim 2$ in combination with thin-layer interferences generates a phase shift between the incoming and outgoing electromagnetic waves. Therefore the periodic electromagnetic acceleration and deceleration of the propagating electrons in the optical field cycles before and after the membrane do not cancel out after passage through the laser focus, as it would happen in free space ${ }^{13}$. In the end there remains a time-dependent overall momentum kick that is proportional to the change of vector potential at the membrane and therefore dependent on the optical phase. In contrast to metal foils ${ }^{13,14}$ or nanostructures ${ }^{15}$, a dielectric membrane has negligible linear or nonlinear optical absorption and can therefore sustain an extreme level of power and fields, enabling strong/effective compression and metrology.

In the experiment, we let the electron beam $(0.48 \times$ speed of light $)$ and the laser beam ( $\mathrm{p}$ polarized, $\sim 15 \mathrm{~mW}$, peak field $\sim 5 \times 10^{7} \mathrm{~V} / \mathrm{m}$ ) hit the membrane under $35^{\circ}$ and $60^{\circ}$ from the surface normal, respectively. In this geometry (close to Brewster's angle) there is predominantly a periodic acceleration and deceleration of the electrons in dependence on the arrival time at the membrane with respect to the laser cycles. Calculations predict ${ }^{16-18}$ that such a periodic energy modulation ${ }^{18}$ can reshape the incoming electron packet after some free-space propagation into a train of attosecond pulses (see Fig. 1). A homogeneous compression of the entire electron beam (diameter $135 \mu \mathrm{m}$ ) is achieved by setting the optical focus larger (diameter $\sim 300 \mu \mathrm{m}$ ) and the pulses longer in time ( 1.7 ps). For temporal pulse characterization, we apply at $3.7 \mathrm{~mm}$ distance a second dielectric membrane (60 nm of silicon) and a second laser beam ("excitation" in Fig. 1). The optical peak field at the silicon membrane is several times higher $\left(\sim 2 \times 10^{8} \mathrm{~V} / \mathrm{m}\right)$ and the foil's refractive index is greater $(\sim 3.5)$. Therefore the previously negligible sideways forces are now substantial and create a time-dependent beam deflection, i.e. a cathode ray oscilloscope at optical frequencies $(\mathrm{PHz})$. Further modulations in time are now irrelevant. Because the sub-cycle 
electron pulse train generated at the first membrane is temporally synchronized to the characterization laser's optical cycles ${ }^{16}$, each attosecond electron pulse in the pulse train sees the same kind of deflection dynamics, and a time-integrated measurement characterizes the average pulse duration and shape ${ }^{1}$.

Figure 2a shows the electron beam shape when all lasers are off. Figure $2 b$ depicts the beam when the characterization oscilloscope ("excitation" in Fig. 1) is activated. We observe an elongated spot with two maxima at the ends (Fig. 2b), which can be identified as the turning points of the periodic deflection at the optical frequency. We infer a streaking speed of $\sim 0.2 \mathrm{mrad} / \mathrm{fs}$, which is $\sim 25$ times faster than previously achieved with $\mathrm{THz}$ radiation ${ }^{13}$. When the compression laser is also activated and adjusted to proper field strength, the streak of Fig. 2b turns into a localized spot that moves up and down with a sinusoidal dependence on the optical phase delay between the two laser beams (see Fig. 2c). This real-space streaking result directly demonstrates in probably the clearest possible way the presence of electron pulses with suboptical-cycle localization in time, for which there has been rather indirect evidence so $\operatorname{far}^{17-19}$.

The duration of the laser-compressed electron pulses can be inferred from the streaked spot-width divided by the streaking speed ${ }^{13}$, but numerically fitting the entire deflectogram to a model is more accurate. We assume a Gaussian electron pulse shape of width $\tau_{e l}$ on top of a time-independent background originating from uncompressed electrons from the non-converging optical cycles ${ }^{16}$. The deflection is modelled by a sinusoidal oscillation with free amplitude and phase at the fixed laser-cycle period of 3.44 fs. Optimization of these four parameters results in the blue lines in Fig. 2d, in noteworthy agreement with the experiment (dots). Figure 2e shows the electron pulse shape in time and reveals a pulse duration (above the background) of $0.35 \mathrm{fs}$ root-mean-square or $0.82 \mathrm{fs}$ full-width-at-half-maximum. These values are upper limits, because potential short-time interferometric instabilities in the experiment cannot entirely be excluded. Figure $2 \mathrm{f}$ shows the electron pulse duration in dependence on the compression laser's peak field strength, showing a characteristic shortening (compression) and subsequently an increase (overcompression) of the pulse width ${ }^{13,20}$. The best-compressed electron pulses in the train are about 35 times shorter than previously reported for atomic-resolution diffraction ${ }^{20}$. 
Beam quality is not substantially altered by the compression. Figures $2 \mathrm{~g}$ reports magnetic waist scans as a measurement of emittance, i.e. product of beam size and divergence. The beam's transverse and longitudinal coherence lengths, which define an electron's ability to interfere with itself when diffracted from a complex material, are directly related ${ }^{21}$. In the $y$ direction (perpendicular to the laser polarization) there is no measurable change of emittance $\left(\epsilon_{p s}^{y} \approx \epsilon_{a s}^{y} \approx 0.94 \mathrm{~nm}\right)$ and in $x$ direction we observe a slight increase from $\epsilon_{p s}^{x} \approx 0.85 \mathrm{~nm}$ to $\epsilon_{a s}^{x} \approx 1.37 \mathrm{~nm}$. This difference originates from tiny sideways deflections at the compressor stage in combination with a non-perfectly collimated electron beam there, an effect that can be avoided at appropriate incidence angles (see Fig. 2h). With $\epsilon_{a s}^{x} \approx 1.37 \mathrm{~nm}$, the predicted transverse coherence for a beam with radius $\sigma_{x} \approx 100 \mu \mathrm{m}$ is $\lambda_{e l} \sigma_{x} /\left(2 \pi \varepsilon_{a s}^{x}\right) \approx 50 \mathrm{~nm}$ and therefore larger than a complex molecule or unit cell. Figure 3 a shows the inner part of a diffraction pattern of 60 nm-thick single-crystalline silicon in $[-1 / \sqrt{2}, \sqrt{2}, 1 / \sqrt{2}]$ direction, obtained with the attosecond electron pulses. Tens of Bragg spots are discernible and demonstrate the atomic resolution. Figure $3 \mathrm{~b}$ shows the rocking curve (angle-dependent intensity) of the $1 \overline{1} 3$ spot, revealing a width that is limited by sample thickness ( $\sim 70 \mathrm{~nm}$ in beam direction) and almost identical traces for uncompressed and compressed electron pulses. The asymmetry is a sign of substantial multiplescattering interferences, but equally strong in both cases. The attosecond pulses do not obtain any substantial energy spread $\Delta E$. The time-energy uncertainty principle and the measured Bragg spot width changes by the compression restrict $\Delta E$ to $4.5-60 \mathrm{eV}$ and $\Delta E / E_{e l}$ to $10^{-4}-10^{-5}$. This monochromaticity is $\sim 100$ times better than in high-harmonic pulses or re-colliding electrons and might be useful for attosecond electron energy loss spectroscopy (EELS) of, for example, innershell dynamics in atoms or some high-energy plasmonic processes. Figure $3 \mathrm{c}$ shows that the overall intensity of the Bragg spots, i.e. the structure factor representing the scattering potential within the unit cell, does not change beyond the experimental shot-noise limit when activating the compression. Diffractive imaging of laser-driven electronic motion in molecules or condensed matter requires percent-level sensitivities to intensity changes ${ }^{22-25}$, a signal-to-noise ratio that our attosecond electron pulses can provide (see Fig. 3c), even although our electron source was operated far from optimum conditions (see methods). These results demonstrate that laser-compression of an electron beam into attosecond pulses does not substantially modify any 
spatial beam parameter; only the effective current is approximately halved by the presence of a constant background (see Fig. 2e) and somewhat further reduced by scattering and absorption at the compressor membrane. In summary, the experimental tools are now available for analytic attosecond-Angstrom diffraction with fundamental space-time resolution.

We quickly report two proof-of-principle applications. Many photoelectric processes in atoms, molecules, solids or liquids occur with subtle delays on attosecond time scales ${ }^{7,26-30}$ which are related to tunnelling or propagation of quasi-free electrons in the potential or band structure of the material, modified by electron-electron interactions and screening. Here we investigate whether Bragg diffraction, i.e. the conversion of crystal momentum to electron momentum, takes time or not. The silicon membrane was oriented for diffraction and simultaneously laser-excited. In this geometry, the electromagnetic streaking dynamics of the attosecond electron wavepackets in the laser field before and after the foil superimposes with the Bragg condition inside the foil, causing diffraction and deflection in synchrony. All Bragg spots show a pronounced timedependent streaking when scanning the laser phase delay (see Fig. 3d). A centre-of-mass analysis $^{26,30}$ reveals any potential delays between a Bragg spot and the direct beam $(000$-spot) with extreme precision (see Fig. 3e). Figure $3 \mathrm{f}$ summarizes the results: Electron-crystal scattering at tens of $\mathrm{keV}$ occurs with no delay on attosecond time scales ( $<40$ as for every single Bragg spot, $<10$ as root-mean-square for all eight spots). Multiple scattering, plasmonic losses, innershell processes, electron-electron exchange interactions or electromagnetic fields exceeding $10^{8}$ $\mathrm{V} / \mathrm{m}$ or $0.3 \mathrm{~T}$ do not affect the attosecond-level timing with which Bragg electrons are emitted from a crystal.

In a second application, we invoke real-space electron microscopy and report a proof-ofprinciple demonstration at low magnification. While the motion of bound electrons occurs naturally on atomic dimensions, where diffraction is an applicable approach for imaging, collective carrier dynamics and transport often involve nanometre dimensions and above, for example in metamaterials or biological/technological light-energy conversions. We illuminated a $130 \times 150-\mu \mathrm{m}$ big silicon membrane with the electron pulse train and adjusted the excitation laser slightly off-angle with the compression laser, in order to generate a travelling optical excitation wave on the sample. Figure 4a shows the membrane's static image and Figure $4 \mathrm{~b}$ reveals the 
time-dependent intensity changes observed with the attosecond pulses; see also supplementary video 1. Fitting a temporal phase to each location reveals the delay map depicted in Fig. 4c, showing a continuous and substantial phase advance from left to right ( $x$ direction). Figure $4 \mathrm{~d}$ illustrates the contrast mechanism: Each point of the sample deflects the electron beam with a phase delay and amplitude that is determined by the optical waveform at that location. Deflection angles $\alpha(x, t)$ originating from the tilted incidence of the excitation wave can be convergent or divergent in $x$, leading to local intensity increases and decreases, which follow in space and time the travelling-wave propagation (see Fig. 4d). A condition for such combined spatial/angular imaging $^{31}$ in the experiment is an out-of-focus magnification (see Fig. 4e); otherwise any ray originating from a certain sample location would end up at the same detector position regardless of deflection. Integrating over $y= \pm 20 \mu \mathrm{m}$ reveals the space-time results of Fig. $4 \mathrm{f}$ in comparison to a simulation based on ray optics and $\alpha(x, t)$ from a travelling wave. While in the middle region the intensity is highest at times where $d \alpha / d x$ is maximum (converging rays, see Fig. 4d), the highest or lowest intensities are observed at the membrane edges $( \pm 64 \mu \mathrm{m})$ at times where $\alpha$ is highest or lowest, respectively. Indeed, the phase shift of the edge features is close to $\pi / 2$ (see Fig. 4f, right panel). Figure 4g shows the $y$-integrated phases along $x$, revealing the travellingwave parameter $(12 \mathrm{as} / \mu \mathrm{m})$, the phase shift at the edges and a $\sim 10$-as accuracy of this proof-ofprinciple microscopy experiment. State-of-the-art instrumentation ${ }^{18}$ and appropriate waveform electron microscopy ${ }^{31}$ with our attosecond pulses will reveal nanoscale and potentially smaller electric and magnetic near-fields with spatial, temporal and vectorial resolution, hence the entire nanophotonic information.

Electron pulses at tens or hundreds of $\mathrm{keV}$ can in principle have multi-keV bandwidths and therefore zeptosecond duration. In our experiment, the degree of compression is limited by the available peak field strength at the compression membrane, by the distance to the sample, by the nonlinear shape of the optical cycles and by the uncorrelated energy spread of the incoming electron packet $(\sim 0.5 \mathrm{eV})$. If these parameters are improved, even shorter electron pulses could be obtained, which might be useful to study electron-electron interactions on sub-attosecond time scales. While pulse trains may offer space-time access to cycle-reversible processes (e.g. linear/nonlinear optics/plasmonics at atoms/molecules/nanostructures, Bloch oscillations, 
tunnelling in laser fields, cycle-induced macroscopic currents) or such with $<$ few-fs time constants (e.g. Auger decays, multi-electron ionization, damping in plasmonics), certain experiments on cycle-irreversible/slower dynamics or processes that cannot be multi-cycle excited due to damage (e.g. dielectric breakdown, molecular dissociations) will require isolated electron pulses and not a pulse train. The reported concept offers several ways to do so. First, the incoming electron packet can be pre-compressed by terahertz radiation ${ }^{13}$ or other means to fewfemtosecond duration, i.e. short enough to inject it into a single cycle of the compression laser. Alternatively, optical single-cycle pulses can be used to deflect an isolated electron pulse out of the pulse train. It is also possible to filter out via single-cycle sideways deflection an attosecond electron pulse directly out of longer pulses or even out of a continuous beam. With compact multi-electron sources and/or sub-MHz laser repetition rates (see methods), about $10^{6}-10^{8}$ e/s will impinge on the sample, enough for pump-probe experiments. Any future advances in source technology will directly apply.

Modern electron microscopy and diffraction have a huge application range and almost no sample-type or morphology restrictions. We therefore think that attosecond electron diffraction and microscopy may become a versatile alternative to conventional attosecond spectroscopy for visualizing fundamental light-matter interactions in space and time.

\section{Acknowledgements}

This work was supported by the European Research Council (grant DIVI) and the Munich-Centre for Advanced Photonics. YM acknowledges support from a JSPS Postdoctoral Fellowship for Research Abroad. We thank Bo-Han Chen and Andrey Ryabov for help with the laser, Simon Stork for help with the foils and Ferenc Krausz for awesome support and inspiring discussions.

\section{Contributions}

YM and PB conceived the experiment, YM measured the data, YM and PB evaluated the data and $\mathrm{YM}$ and $\mathrm{PB}$ wrote the manuscript. 


\section{Competing financial interests}

The authors declare no competing financial interests. 


\section{References}

[1] P. M. Paul, E. S. Toma, P. Breger, G. Mullot, F. Auge, P. Balcou, H. G. Muller, and P. Agostini. Observation of a train of attosecond pulses from high harmonic generation. Science, 292(5522):1689-1692, June 2001.

[2] M. Hentschel, R. Kienberger, C. Spielmann, G. A. Reider, N. Milosevic, T. Brabec, P. Corkum, U. Heinzmann, M. Drescher, and F. Krausz. Attosecond metrology. Nature, 414(6863):509-513, November 2001.

[3] R. Kienberger, E. Goulielmakis, M. Uiberacker, A. Baltuska, V. Yakovlev, F. Bammer, A. Scrinzi, T. Westerwalbesloh, U. Kleineberg, U. Heinzmann, M. Drescher, and F. Krausz. Atomic transient recorder. Nature, 427(6977):817-821, February 2004.

[4] O. Smirnova, Y. Mairesse, S. Patchkovskii, N. Dudovich, D. Villeneuve, P. Corkum, and M. Y. Ivanov. High harmonic interferometry of multi-electron dynamics in molecules. Nature, 460(7258):972-977, August 2009.

[5] G. Sansone, F. Kelkensberg, J. F. Perez-Torres, F. Morales, M. F. Kling, W. Siu, O. Ghafur, P. Johnsson, M. Swoboda, E. Benedetti, F. Ferrari, F. Lepine, J. L. SanzVicario, S. Zherebtsov, I. Znakovskaya, A. L’Huillier, M. Y. Ivanov, M. Nisoli, F. Martin, and M. J. J. Vrakking. Electron localization following attosecond molecular photoionization. Nature, 465(7299):763-U3, June 2010.

[6] M. Lucchini, S. A. Sato, A. Ludwig, J. Herrmann, M. Volkov, L. Kasmi, Y. Shinohara, K. Yabana, L. Gallmann, and U. Keller. Attosecond dynamical Franz-Keldysh effect in polycrystalline diamond. Science, 353(6302):916-919, August 2016.

[7] Z. S. Tao, C. Chen, T. Szilvasi, M. Keller, M. Mavrikakis, H. Kapteyn, and M. Murnane. Direct time-domain observation of attosecond final-state lifetimes in photoemission from solids. Science, 353(6294):62-67, July 2016.

[8] H. Niikura, F. Legare, R. Hasbani, A. D. Bandrauk, M. Y. Ivanov, D. M. Villeneuve, and P. B. Corkum. Sub-laser-cycle electron pulses for probing molecular dynamics. Nature, 417(6892):917-922, June 2002.

[9] J. Itatani, J. Levesque, D. Zeidler, H. Niikura, H. Pepin, J. C. Kieffer, P. B. Corkum, and D. M. Villeneuve. Tomographic imaging of molecular orbitals. Nature, 432(7019):867871, December 2004.

[10] C. I. Blaga, J. L. Xu, A. D. DiChiara, E. Sistrunk, K. K. Zhang, P. Agostini, T. A. Miller, L. F. DiMauro, and C. D. Lin. Imaging ultrafast molecular dynamics with laser-induced electron diffraction. Nature, 483(7388):194-197, March 2012. 
[11] B. Wolter, M. G. Pullen, A.-T. Le, M. Baudisch, K. Doblhoff-Dier, A. Senftleben, M. Hemmer, C. D. Schröter, J. Ullrich, T. Pfeifer, R. Moshammer, S. Gräfe, O. Vendrell, C. D. Lin, and J. Biegert. Ultrafast electron diffraction imaging of bond breaking in diionized acetylene. Science, 354(6310):308-312, 2016.

[12] W. Schneider, A. Ryabov, Cs. Lombosi, T. Metzger, Zs. Major, J. A. Fulop, and P. Baum. 800-fs, 330-Â $\mu \mathrm{J}$ pulses from a 100-W regenerative $\mathrm{Yb}: Y A G$ thin-disk amplifier at $300 \mathrm{kHz}$ and THz generation in LiNbO3. Optics Letters, 39(23):6604-6607, December 2014.

[13] C. Kealhofer, W. Schneider, D. Ehberger, A. Ryabov, F. Krausz, and P. Baum. All-optical control and metrology of electron pulses. Science, 352(6284):429-433, April 2016.

[14] F. O. Kirchner, A. Gliserin, F. Krausz, and P. Baum. Laser streaking of free electrons at 25 keV. Nature Photonics, 8(1):52-57, January 2014.

[15] B. Barwick, D. J. Flannigan, and A. H. Zewail. Photon-induced near-field electron microscopy. Nature, 462(7275):902-906, December 2009.

[16] P. Baum and A. H. Zewail. Attosecond electron pulses for 4D diffraction and microscopy. Proceedings of the National Academy of Sciences of the United States of America, 104(47):18409-18414, November 2007.

[17] C. M. S. Sears, E. Colby, R. Ischebeck, C. McGuinness, J. Nelson, R. Noble, R. H. Siemann, J. Spencer, D. Walz, T. Plettner, and R. L. Byer. Production and characterization of attosecond electron bunch trains. Physical Review Special Topics-accelerators and Beams, 11(6):061301, June 2008.

[18] A. Feist, K. E. Echternkamp, J. Schauss, S. V. Yalunin, S. Schaefer, and C. Ropers. Quantum coherent optical phase modulation in an ultrafast transmission electron microscope. Nature, 521(7551):200, May 2015.

[19] M. Kozak, J. McNeur, K. J. Leedle, H. Deng, N. Schonenberger, A. Ruehl, I. Hartl, J. S. Harris, R. L. Byer, and P. Hommelhoff. Optical gating and streaking of free electrons with sub-optical cycle precision. Nature Communications, 8:14342, January 2017.

[20] A. Gliserin, M. Walbran, F. Krausz, and P. Baum. Sub-phonon-period compression of electron pulses for atomic diffraction. Nature Communications, 6:8723, October 2015.

[21] W. J. Engelen, M. A. van der Heijden, D. J. Bakker, E. J. D. Vredenbregt, and O. J. Luiten. High-coherence electron bunches produced by femtosecond photoionization. Nature Communications, 4:1693, April 2013.

[22] H. C. Shao and A. F. Starace. Detecting electron motion in atoms and molecules. Physical Review Letters, 105(26):263201, December 2010. 
[23] V. S. Yakovlev, M. I. Stockman, F. Krausz, and P. Baum. Atomic-scale diffractive imaging of sub-cycle electron dynamics in condensed matter. Scientific Reports, 5:14581, September 2015.

[24] J. Stingl, F. Zamponi, B. Freyer, M. Woerner, T. Elsaesser, and A. Borgschulte. Electron transfer in a virtual quantum state of LiBH4 induced by strong optical fields and mapped by femtosecond x-ray diffraction. Physical Review Letters, 109(14):147402, October 2012.

[25] Y. Morimoto, R. Kanya, and K. Yamanouchi. Light-dressing effect in laser-assisted elastic electron scattering by Xe. Physical Review Letters, 115(12):123201, September 2015.

[26] A. L. Cavalieri, N. Mueller, Th. Uphues, V. S. Yakovlev, A. Baltuska, B. Horvath, B. Schmidt, L. Bluemel, R. Holzwarth, S. Hendel, M. Drescher, U. Kleineberg, P. M. Echenique, R. Kienberger, F. Krausz, and U. Heinzmann. Attosecond spectroscopy in condensed matter. Nature, 449(7165):1029-1032, October 2007.

[27] P. Eckle, A. N. Pfeiffer, C. Cirelli, A. Staudte, R. Dorner, H. G. Muller, M. Buttiker, and U. Keller. Attosecond ionization and tunneling delay time measurements in helium. Science, 322(5907):1525-1529, December 2008.

[28] M. Schultze, M. Fiess, N. Karpowicz, J. Gagnon, M. Korbman, M. Hofstetter, S. Neppl, A. L. Cavalieri, Y. Komninos, Th. Mercouris, C. A. Nicolaides, R. Pazourek, S. Nagele, J. Feist, J. Burgdoerfer, A. M. Azzeer, R. Ernstorfer, R. Kienberger, U. Kleineberg, E. Goulielmakis, F. Krausz, and V. S. Yakovlev. Delay in photoemission. Science, 328(5986):1658-1662, June 2010.

[29] K. Klunder, J. M. Dahlstrom, M. Gisselbrecht, T. Fordell, M. Swoboda, D. Guenot, P. Johnsson, J. Caillat, J. Mauritsson, A. Maquet, R. Taieb, and A. L'Huillier. Probing single-photon ionization on the attosecond time scale. Physical Review Letters, 106(14):143002, April 2011.

[30] S. Neppl, R. Ernstorfer, A. L. Cavalieri, C. Lemell, G. Wachter, E. Magerl, E. M. Bothschafter, M. Jobst, M. Hofstetter, U. Kleineberg, J. V. Barth, D. Menzel, J. Burgdorfer, P. Feulner, F. Krausz, and R. Kienberger. Direct observation of electron propagation and dielectric screening on the atomic length scale. Nature, 517(7534):342-346, January 2015.

[31] A. Ryabov and P. Baum. Electron microscopy of electromagnetic waveforms. Science, 353(6297):374-377, July 2016. 
Figure 1 | Concept and experiment. A photo-emitted picosecond electron pulse (blue) with a sub-atomic de Broglie wavelength is temporally modulated at a dielectric foil (green) by the optical cycles of a femtosecond laser (red) into a train of attosecond electron pulses (blue). Electron diffraction from a cycle-excited sample (green) can produce atomic resolution in space and time. Alternatively, real-space electron microscopy can reveal optical near-field vectors and phase delays with sub-cycle time resolution.

Figure 2 | Attosecond electron pulses. a, Basic electron beam profile. b, Streaking deflection of long electron pulses by optical field cycles. c, Streaking deflection of laser-compressed electron pulses in dependence of delay. The oscillatory shape demonstrates the presence of sub-opticalcycle electron pulses. The streaking widths at the zero-crossings indicate the pulse duration. d, Comparison of experiment (dots) and numerical simulation (blue lines). e, Electron pulse shape, evaluated directly from the raw data (dots) or via the simulation fit (blue). The electron pulse duration (above background) is 820 as (full width, see black arrow) or 350 as (root-mean-square). f, Electron pulse duration in dependence on the compression laser's peak field strength $E_{p e a k} . \mathbf{g}$, Beam quality measurements (emittance) for picosecond pulses (black) in comparison to the attosecond pulse train (blue). h, Numerical simulation (finite difference time domain, Lumerical Inc.) of the compression efficiency ( $\|$ ) and residual sideways deflection $(\stackrel{\perp}{)}$ ) in dependence on the laser-electron angle ( $\left.\alpha_{\text {laser-e }}\right)$ for a fixed electron-foil incidence of $35^{\circ}$ and a peak field strength of $10^{8} \mathrm{~V} / \mathrm{m}$. An angle of $\sim 40^{\circ}$ provides efficient compression without sideways deflection.

Figure 3 | Atomic diffraction with attosecond electron pulses. a, Diffraction pattern of singlecrystalline silicon, taken with the attosecond electron pulse train. The not labelled Bragg spots are forbidden but visible via multiple scattering. b, Rocking curve of the $1 \overline{1} 3$ spot for picosecond pulses in comparison to the attosecond electron pulse train. Inset, crystal structure of silicon. c, Absolute intensity of all Bragg spots, measured with the attosecond pulses and normalized to the picosecond case. Error bars denote the shot noise of the measurement ( $\sim 140 \mathrm{~s}$ integration). d, Streaking deflectograms of three example Bragg spots; all of them behave similarly. e, Centre-ofmass of the deflectogram of the $1 \overline{1} 3$ spot vs. the direct beam for delay analysis. Inset, illustration of delays in Bragg spot emission. f, Measured attosecond-level delay of different Bragg spot 
emissions with respect to the direct beam. The filled and open circles are the results of centre-ofmass analysis and deflectogram fit, respectively. Error bars represent standard deviation. The average delay is $(4.6 \pm 5.4)$ as.

\section{Figure 4 | Attosecond electron microscopy of electromagnetic waveform propagation. a,} Shadow image of a $60-\mathrm{nm}$ thick silicon membrane (grey) with $\mathrm{SiN} / \mathrm{SiO}$ overcoat (black) at low magnification. The excitation field's polarization direction $\mathrm{E}(\mathrm{t})$ is indicated by the arrow. $\mathbf{b}$, Image changes in dependence on time delay. The arrows indicate the polarization direction of the excitation fields. $\mathbf{c}$, Phase map of the measured intensity oscillations for every position on the membrane. d, Illustration of how a travelling-wave excitation (red) produces converging and diverging electron trajectories (blue) of the attosecond pulse train. e, Illustration of the imaging geometry with an image plane slightly off the detector in order to observe positions and angles at the same time (differential phase contrast). f, Position-dependent intensity changes vs. time in comparison to a trajectory simulation. $\mathbf{g}$, Phase and delay across the sample (dots) in comparison to the simulation (blue). The travelling wave advances in space-time by 12 as $/ \mu \mathrm{m}$.

Supplementary Video 1 | Attosecond electron microscopy of a traveling wave. The left panel shows the raw microscopic image of the silicon window in time. The right panel shows the change of the images with respect to the excitation delay. The scale bars represent $100 \mu \mathrm{m}$. 


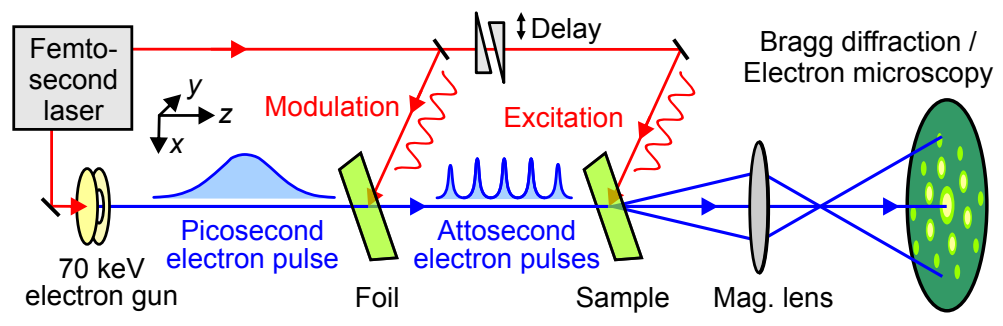

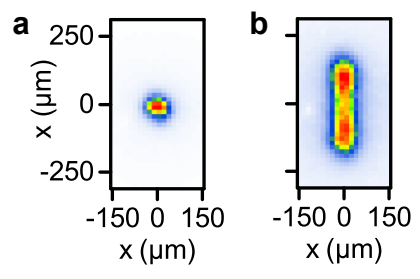
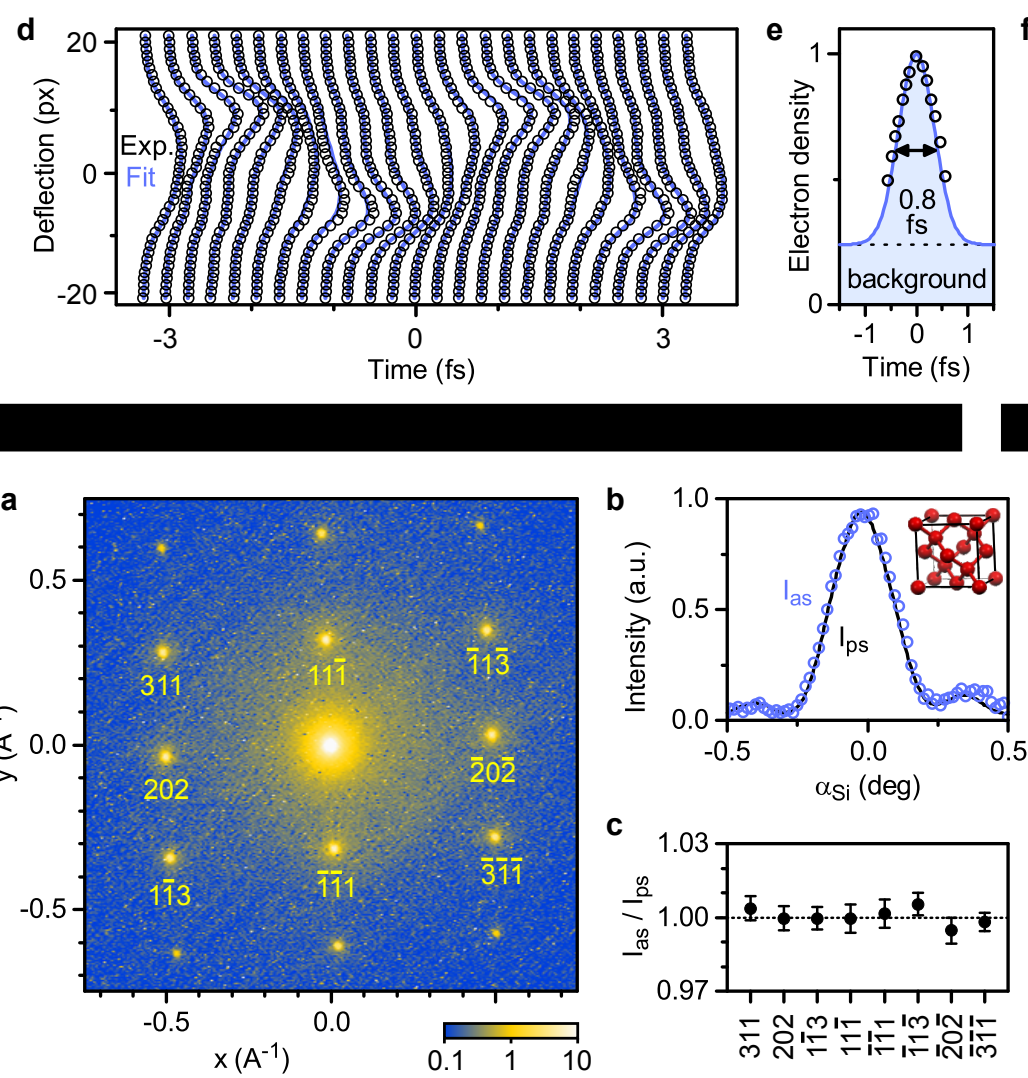
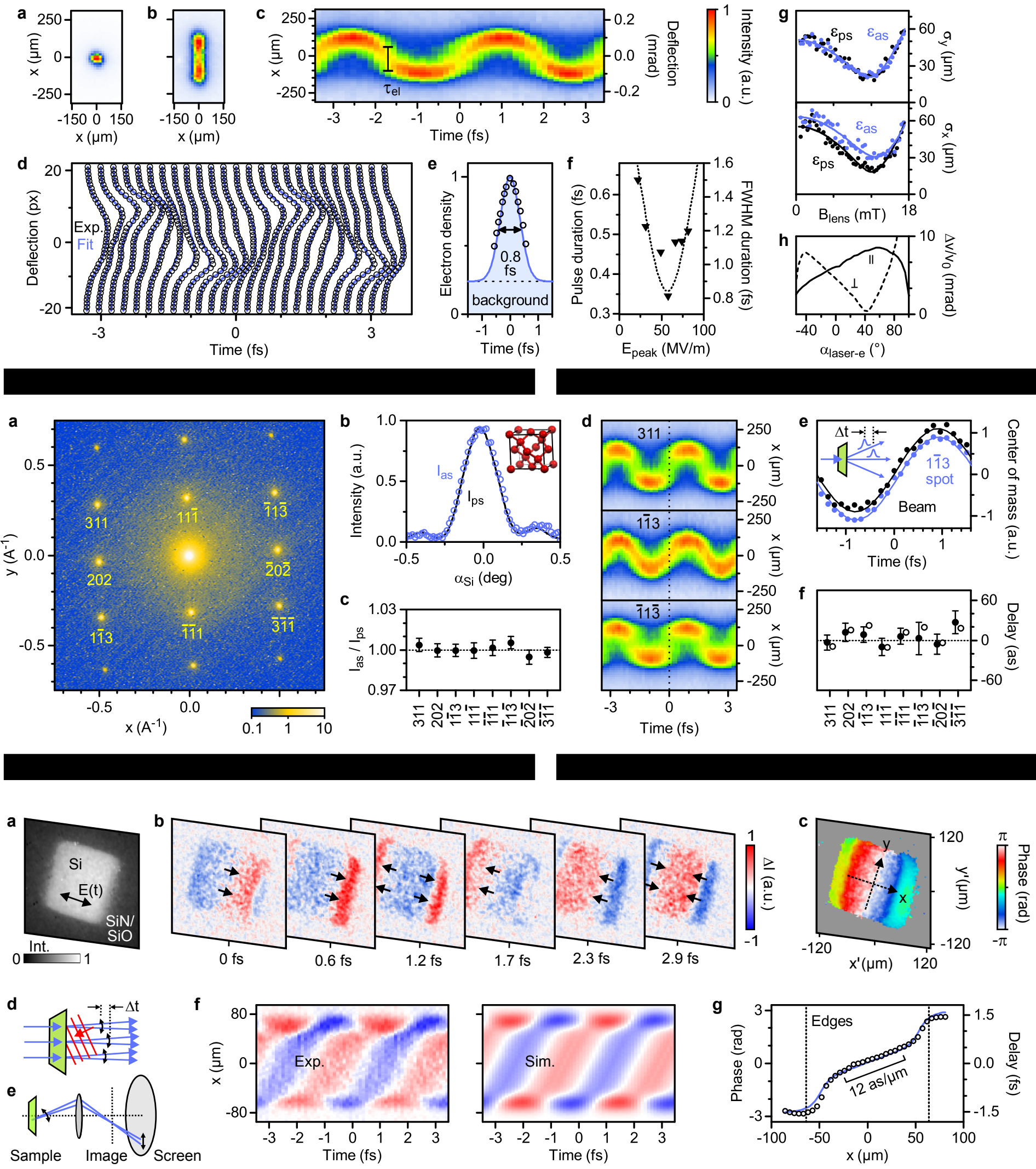


\title{
Diffraction and microscopy with attosecond electron pulse trains
}

\author{
Y. Morimoto and P. Baum
}
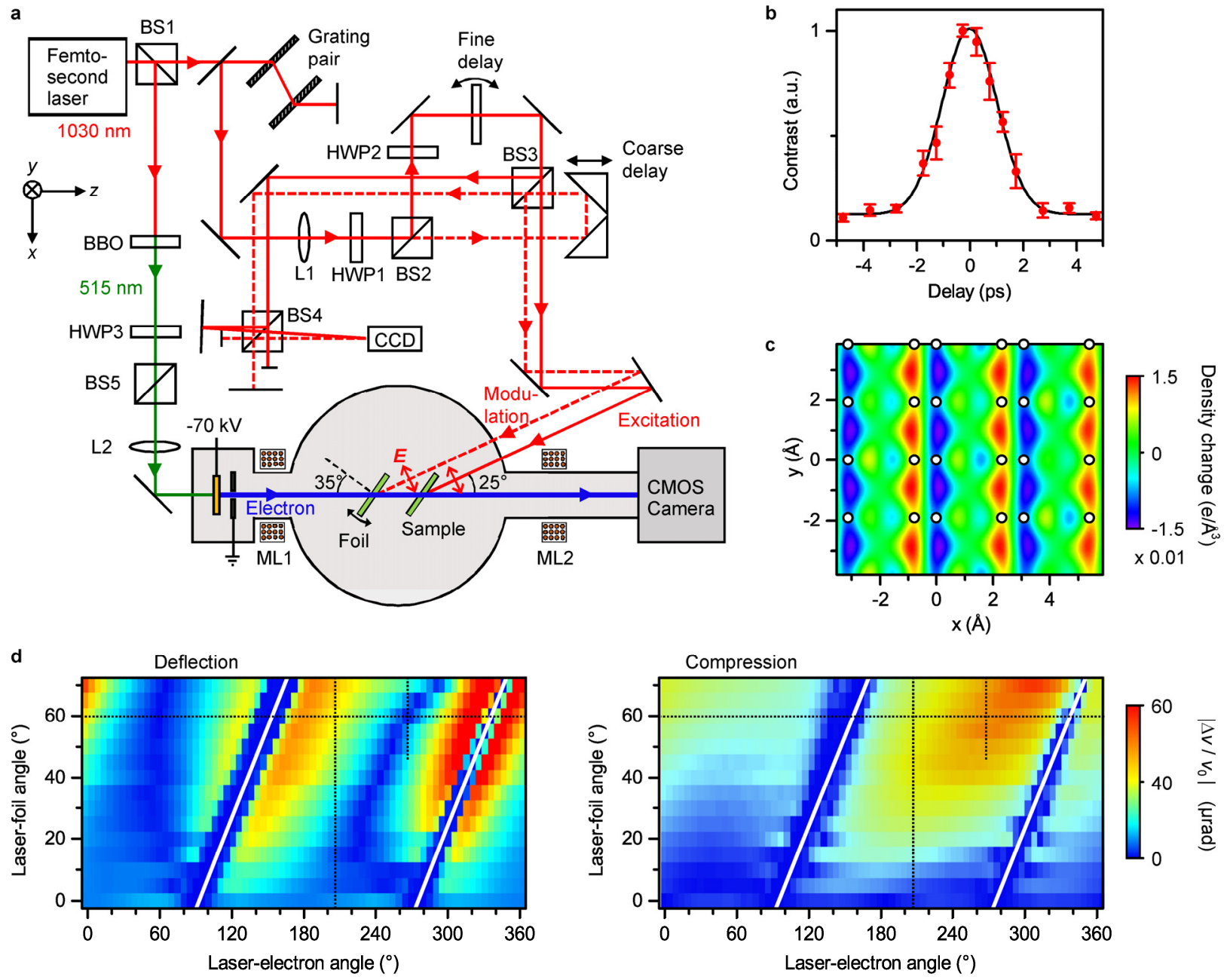

Figure S1 | Details of the experimental setup and field-driven electron motion in Silicon.

a, Setup and beam paths. Red: laser beam (1030 nm wavelength), green: second-harmonic beam (515 nm wavelength), blue: electron beam, yellow: gold photocathode, BS: beam splitter, BBO: $\beta$-barium-borate crystal, HWP: half-wave plate, L: lens, ML: magnetic lens, CCD: optical beam profiling camera. $\mathbf{b}$, Delay-dependence of the contrast of the electron deflectogram when scanning the two laser pulses. Red circles: experimental result. Black curve: fitting curve. c, Numerical simulation of a possible electric-field-induced change of the charge density in $\mathrm{Si}$, as seen by the electron beam and averaged along the propagation direction. The small circles represent the position of Si nuclei. d, Angle-dependence of streaking deflection (left panel) and temporal compression (right panel). White lines denote impossible configurations, dotted long lines indicate the experimental conditions and the short dotted lines show a compressor setting without deflection. For more information, see methods section. 